\title{
Algunos crinoides (Echinodermata-Crinoidea) del Pérmico de la región de Pemuxco, Hidalgo
}

\author{
Blanca E. Buitrón Sánchez, Oscar Lopez Lara, Daniel Vachard, Andrea Sarai Hernández Barroso
}

Blanca E. Buitrón Sánchez

blancab@unam.mx

Instituto de Geología, Universidad Nacional Autónoma de México, C.P. 04510, CDMX, México.

\section{Oscar López Lara}

Andrea Sarai Hernández Barroso

División de Ingeniería en Ciencias de la Tierra, Facultad de Ingeniería, Universidad Nacional Autónoma de México, C.P. 04510, CDMX, México.

\section{Daniel Vachard}

Laboratorio del Paleozoico, Universidad de Ciencias y Tecnologías de Lille, Francia.
BOL. SOC. GEOL. MEX. 2017

VOL. 69 NO. 1

P. $21-34$

\section{RESUMEN}

Se describen cinco especies de crinoides Cylindrocaulis cusfiski Moore y Jeffords, 1968, Baryschir anosus Moore y Jeffords, 1968, Cyclocaudex jucundus Moore y Jeffords, 1968, Cyclocaudex plenus Moore y Jeffords, 1968, Cycloscapus laevis Moore y Jeffords, 1968 recolectados en afloramientos de la Formación Tuzancoa del Pérmico en la región de Pemuxco, en el estado de Hidalgo, México. La presencia en dichos afloramientos del fusulínido Skinnerella, en el mismo nivel que los crinoides, precisa la edad del Pérmico inferior (Leonardiano) para esta secuencia. Los crinoides procedentes de la región de Pemuxco, probablemente vivieron en un ambiente batial de facies flysch, que corresponde a un depósito rítmico de la Formación Tuzancoa y que sugiere agua relativamente poco profunda, en un ambiente geotectónico inestable.

Los crinoides han sido citados de otras localidades del Paleozoico superior de México (Calnali, Hidalgo; Patlanoaya y Cuxtepeque Puebla; Ixtaltepec, Oaxaca, Cañón de la Peregrina, Tamaulipas, cerros El Tule y Sierra Las Mesteñas, Sonora. En los Estados Unidos de Norteamérica se describieron de Texas (Lutita Mingus, Grupo Strawn; Formación Thrifty, Formación Graham, Caliza Marble Falls) y de Oklahoma (Formación Dornick Hills).

Palabras clave: Crinoidea, Pérmico, Formación Tuzancoa, Hidalgo, México.

\section{ABSTRACT}

From outcrops of the Permian Tuzancoa Formation in the region of Pemuxco, Hidalgo State, México, five species of crinoids are described Cylindrocaulis cusfiski Moore $\mathcal{E}^{\circ}$ Feffords, 1968, Baryschir anosus Moore $\mathcal{E}^{\circ}$ Jeffords, 1968, Cyclocaudex costatus Moore छ Jeffords, 1968, Cyclocaudex jucundus Moore E Feffords, 1968, Cycloscapus laevis Moore E Feffords, 1968. The presence in these outcrops of the Skinnerella fusulinid, on the same level as the crinoids, determines the precise age of the lower Permian sequence (Leonardian) for this sequence.

The crinoids assemblage of Pemuxco region probably lived in a bathyal environment of flysch facies, which correspond ed to a rhythmic deposit present in the Tuzancoa Formation and suggesting a shallow water environment in an unstable geotectonic setting. The occurrence of continental plants remains could indicate that they were transported.

Crinoids have been cited in other parts of the upper Paleozoic of Mexico that includes Calnali, Hidalgo, Puebla Patlanoaya and Cuxtepeque, Ixtaltepec, Oaxaca, Peregrina Canyon, Tamaulipas, and The El Tule and Mesteñas mountain range, Sonora. In the United States they have been reported in Texas (Shale Mingus, Strawn Group, Training Thrifty, Training Graham, and Limestone Marble Falls) and Oklahoma (Training Dornick Hills).

Keywords: Crinoidea, Permian, Tuzancoa Formation, Hidalgo, Mexico. 


\section{Introducción}

Las investigaciones sobre rocas sedimentarias y su biota del Pérmico de México son escasas. Entre las publicaciones referentes a estudios de las rocas del Paleozoico de la región, se encuentra la primera cartografía geológica que abarcó la parte central del Anticlinorio y fue realizada por Von Kuegelgen (1958). Carrillo-Bravo (1959, 1961, 1965) hizo la prospección geológica del Anticlinorio de Huayacocotla, donde estableció la columna estratigráfica y describió la litología de las diferentes formaciones que ahí afloran, entre ellas la Formación Guacamaya del Pérmico y Martínez-Pérez (1962) estudió la geología del área de Zacualtipán-Tianguistengo en Hidalgo. Posteriormente, Ochoa-Camarillo (1996) en su estudio sobre la geología del Anticlinorio de Huayacocotla en la región de Molango, Hidalgo propuso el nombre de Formación Tuzancoa para una secuencia vulcano-sedimentaria conformada por brecha volcánica con intercalaciones de lentes calcáreos con abundantes fusulínidos y crinoides que afloran en la cercanía del poblado de Tuzancoa, Hidalgo.

Particularmente, entre las publicaciones en que se menciona a invertebrados del Paleozoico del Anticlinorio de Huayacocotla, se encuentra la de Pérez-Ramos (1979) quien documentó la existencia de los fusulínidos del Paleozoico Superior procedentes de la región de Tlahualompa, Hidalgo. Brunner (1984) en su catálogo de microfósiles del Paleozoico constata la presencia de fusulínidos. Buitrón et al. (1987) describieron las especies de crinoides procedentes de Calnali, Hidalgo. Ochoa-Camarillo et al. (1997) mencionan la presencia de braquiópodos y crinoides de afloramientos del Paleozoico de la región. Vachard et al. (1997) describieron la estratigrafía y el contenido biótico de localidades paleozoicas de las cercanías de Pemuxco. Rosales-Lagarde et al. (1997) informaron sobre la existencia de fusulínidos y crinoides en esta región. Arellano et al. (1998) analizaron aspectos estratigráficos, estructurales y paleogeográficos de Pemuxco y su contenido biótico y Rosales-Lagarde et al. (2005) propusieron formalmente para esta región una nueva formación que fue denominada Formación Tuzancoa.

\subsection{LOGALIZAGIÓN GEOGRÁFICA}

El estado de Hidalgo limita al norte con el estado de San Luis Potosí, al sur con los estados de México y Tlaxcala, al este con los estados de Veracruz y Puebla y al oeste con el estado de Querétaro; con una superficie total de $20987 \mathrm{~km}^{2}$, representa el $1.06 \%$ de la superficie del país. El área de estudio está comprendida entre las coordenadas $98^{\circ} 27^{\prime}$ a $98^{\circ} 35^{\prime}$ de longitud oeste y $20^{\circ} 36^{\prime}$ a $20^{\circ} 45^{\prime}$ de latitud norte, se encuentra entre los poblados de Pemuxco y Jalapa, en el sector centro-septentrional de la región de Zacualtipán de Ángeles (INEGI, 2001), conforme a una franja de rumbo SE-NW. Los mejores afloramientos están localizados sobre los ríos Tianguistengo y Mimiaguaco. Particularmente, los afloramientos donde fueron recolectados los crinoides se encuentran en una barranca con coordenadas $98^{\circ} 32^{\prime} \mathrm{O}$ y $20^{\circ} 44^{\prime} \mathrm{N}$ (Figura 1).

\subsection{CONSIDERAGIONES ESTRATIGRÁFICAS}

La Formación Guacamaya fue definida inicialmente por Carrillo-Bravo (1961) en el Anticlinorio de Huayacocotla, con localidad tipo en la cercanía de Ciudad Victoria, Tamaulipas. Ochoa-Camarillo (1996) propone el nombre de Formación Tuzancoa para este equivalente de la Formación Guacamaya y sugiere que no obstante de algunas diferencias litológicas entre las formaciones Guacamaya y Tuzancoa, se pueden correlacionar. Posteriormente, fue redefinida como Formación Tuzancoa por Rosales-Lagarde et al. (2005) y aflora en tres regiones a lo largo de la margen este-central del Golfo de México: en el Cañón de La Peregrina, al NW de Ciudad Victoria, Tamaulipas, en la región de Molango, en el estado de Hidalgo y entre los estados de Veracruz y Puebla. Campa y Coney (1983) y Rosales-Lagarde et al. (1997) incluyen esta formación dentro del Terreno Sierra Madre.

En la región de Pemuxco las rocas más antiguas son del Precámbrico y corresponden al Gneis Huizno- 


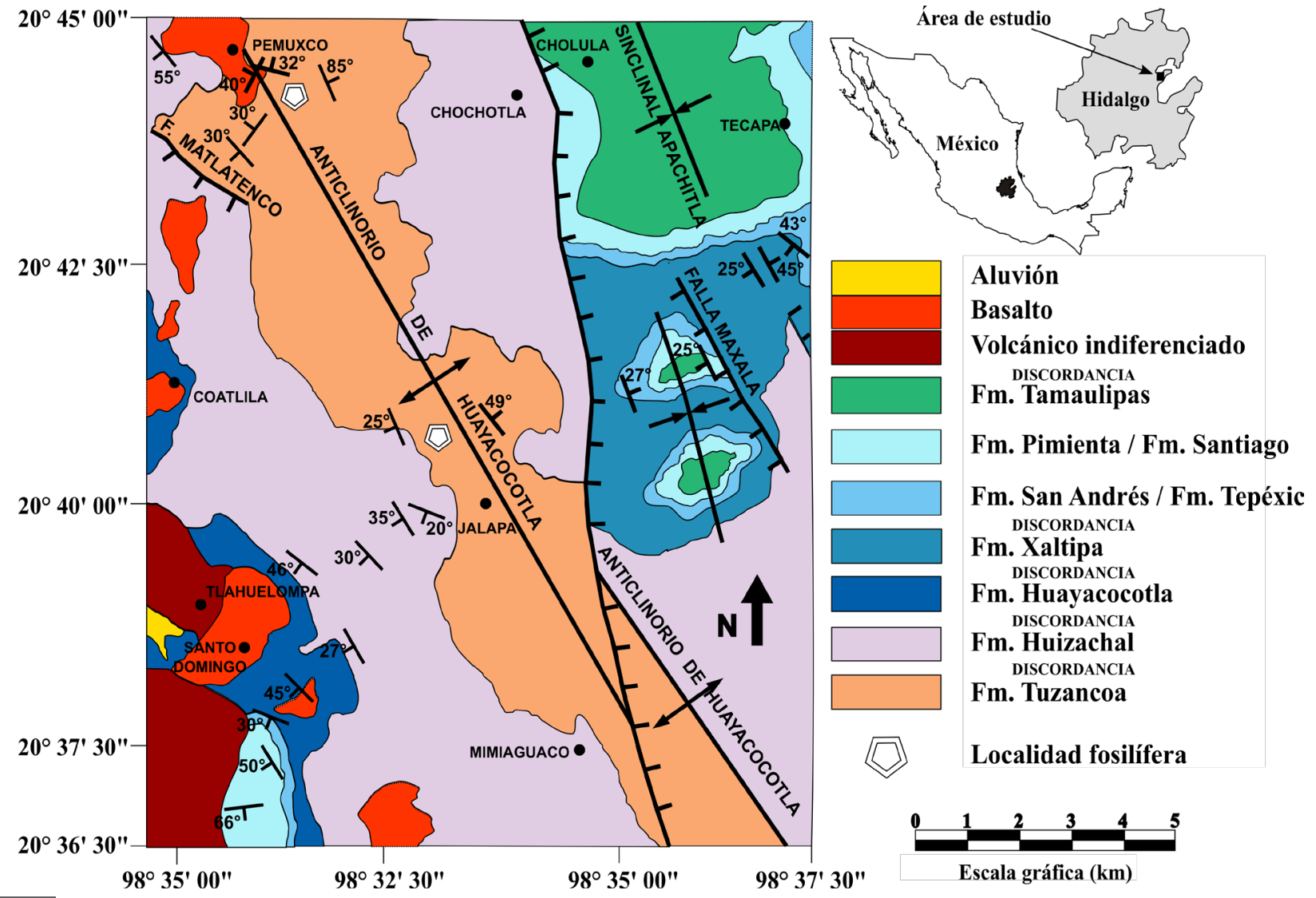

Figura 1 Mapa geológico del área de Pemuxco, Hidalgo (Modificado de Arellano et al. 1998).

palagranulítico de color gris verdoso el cual aflora aproximadamente a $5 \mathrm{~km}$, en las cercanías de los ríos Chinameca y Huiznopala (Carrillo-Bravo, 1959, 1961, 1965; Ochoa-Camarillo et al., 1997; Rosales Lagarde et al., 1997, 2005). Esta unidad soporta discordantemente a la Formación Tuzancoa del Pérmico, con un espesor de 7000 m, asimismo sobreyace discordantemente la Formación Huizachal del Triásico (Ochoa-Camarillo, 1996, fig. 3; Arellano et al., 1998, fig. 1).

Los fósiles de crinoides estudiados fueron recolectados en estratos de la Formación Tuzancoa (Ochoa-Camarillo, 1996, 1997; Rosales-Lagarde, 2005).

\subsection{FORMAGIÓN TUZANGOA}

Las rocas de la Formación Tuzancoa están expuestas en la porción central del Anticlinorio de Huayacocotla y en otros afloramientos, los cuales se encuentran en el arroyo Chipoco, a $4 \mathrm{~km}$, al oeste de Tlanchinol, Hidalgo y en una superficie de aproximadamente $300 \mathrm{~km}^{2}$, que se extiende desde escasos kilómetros al sureste del camino Ixtlahuaco-Tlanchinol hasta el río Chinameca, en el camino Yatipán-Tianguistengo, en el río Tlahualompa y sobre el camino San Mateo-Cholula, Veracruz. En el río Chinameca, la Formación Tuzancoa está conformada por más de $2000 \mathrm{~m}$ de lutita de color negro, en capas de espesor delgado a medio, que alternan con estratos delgados, medianos y gruesos de arenisca de grano medio y grueso a conglomerático. Con frecuencia la arenisca contiene fusulínidos y crinoides, ésta intemperiza a color gris verdoso o café ligeramente rojizo, alternando ocasionalmente con bancos gruesos de conglomerados que contienen abundantes fragmentos de caliza con fusulínidos y crinoides (Ochoa-Camarillo, 1996, 1997; Arellano et al., 1998; Rosales-Lagarde et al., 2005). 
En el camino de Manitla a Mecalapa, al norte del río Chinameca, se encuentran además algunas capas de caliza de color gris con abundantes fusulínidos y corales. Sobre el camino Tianguistengo-Yatipan, Hidalgo, la Formación Tuzancoa está representada por el mismo tipo de sedimentos que en el río Tianguistengo; dentro de esta secuencia al oeste del Rancho Otlamalacatla, se hallan varios horizontes de caliza con fusulínidos y algunas capas de areniscas que contienen restos de tallos de plantas (Ochoa, 1996; Silva, 1987). En el río Tlahualompa, la columna sedimentaria del Pérmico se compone de más de $2000 \mathrm{~m}$ de lutita negra, bandeada, en capas que varían de espesor de unos $5 \mathrm{~cm}$, a más de $2 \mathrm{~m}$, con coloración ocasionalmente gris verdoso. Estas capas alternan con estratos delgados a medianos de arenisca de color gris oscuro y negro. En la región de Calnali y el arroyo de Jalpa, al oeste de Tlanchinol, Hidalgo, la formación está representada por una secuencia rítmica de más de $200 \mathrm{~m}$ de lutita, arenisca y algunos conglomerados de color negro.

Las rocas de la Formación Tuzancoa que afloran en el río Chinameca soportan en discordancia angular a las capas de la Formación Huizachal y está en contacto por falla, con el Gneis Huiznopala de edad precámbrica. Sobre el camino Yatipán-Tianguistengo, Hidalgo, al oeste del poblado de Pemuxco, las rocas pérmicas soportan discordantemente a la Formación Huizachal y al este de Otlamalacatla están cubiertas, también discordantemente, por rocas del Jurásico Superior (Ochoa-Camarillo, 1996). Al norte y noroeste del área, la Formación Tuzancoa, se presenta discordantemente bajo rocas triásicas de la Formación Huizachal y en otros lados descansan capas del Jurásico Superior sobre la Formación Tuzancoa (Ochoa-Camarillo, 1997) . El aspecto rítmico que presentan las rocas de la Formación Tuzancoa, hace pensar en un ambiente de depósito batial de aguas relativamente poco profundas con facies de tipoflysch y en un ambiente geotectónico inestable, aunque la presencia de restos de plantas, pudiera indicar la cercanía a islas o continentes (Vachard et al., 1997; Arellano et al., 1998). La formación contiene abundantes fu- sulínidos (Brunner, 1984), además de briozoarios, crinoides, braquiópodos, pelecípodos y algunos trilobites; también se encuentran horizontes con abundantes fragmentos de tallos y hojas de plantas (Silva, 1987), e icnofósiles (Stinnesbeck, 1994), por lo cual no hubo problema en fijar su edad con base en el contenido fosilífero y su posición estratigráfica, concluyendo así que a la Formación Tuzancoa se le asigna una edad wolfcampiana y posiblemente leonardiana en su cima. Además, por sus características litológicas es correlacionable con los sedimentos pérmicos de la Formación Guacamaya expuestos cerca de Ciudad Victoria (Anticlinorio de Huizachal-Peregrina) en Tamaulipas (Carrillo-Bravo, 1965; Moreno-Cano y Patiño Ruiz, 1981; Ochoa-Camarillo, 1996; Arellano et al., 1998).

\subsection{ESTRATIGRAFÍA LOGAL DEL ÁREA DE PEMUXCO, HIDALGO}

En la región de Pemuxco se encuentra expuesta una columna sedimentaria de aproximadamente $7000 \mathrm{~m}$ de espesor, constituida por las formaciones Tuzancoa, Huizachal, Huayacocotla, Xaltipa, San Andrés/Tepexic, Santiago, Pimienta y Tamaulipas. En este trabajo solamente se hace hincapié en la Formación Tuzancoa, donde fue recolectado el material fósil del estudio. En la región de Pemuxco se midió una sección de aproximadamente 1081 m de la Formación Tuzancoa, que aflora sobre el río Tianguistengo, antes de su confluencia con el río Amajac, en la proximidad del área estudiada se encuentra el Gneis Huiznopala, esta unidad presenta contacto discordante con la Formación Tuzancoa y el contacto superior de esta última formación es discordante con los lechos rojos de la Formación Huizachal. Dentro de la secuencia sedimentaria medida se reconocieron 14 unidades de la cima a la base y son las siguientes (Figura 2): Unidad A: Secuencia turbidítica en estratos entre 10 y $30 \mathrm{~cm}$ de espesor, con secuencias Bouma completas, la fracción arenosa es de color café claro y oscuro en tanto que la fracción pelítica es de color gris oscuro. La unidad presenta algunos lentes de arenisca y tiene un espesor total de $74 \mathrm{~m}$. 


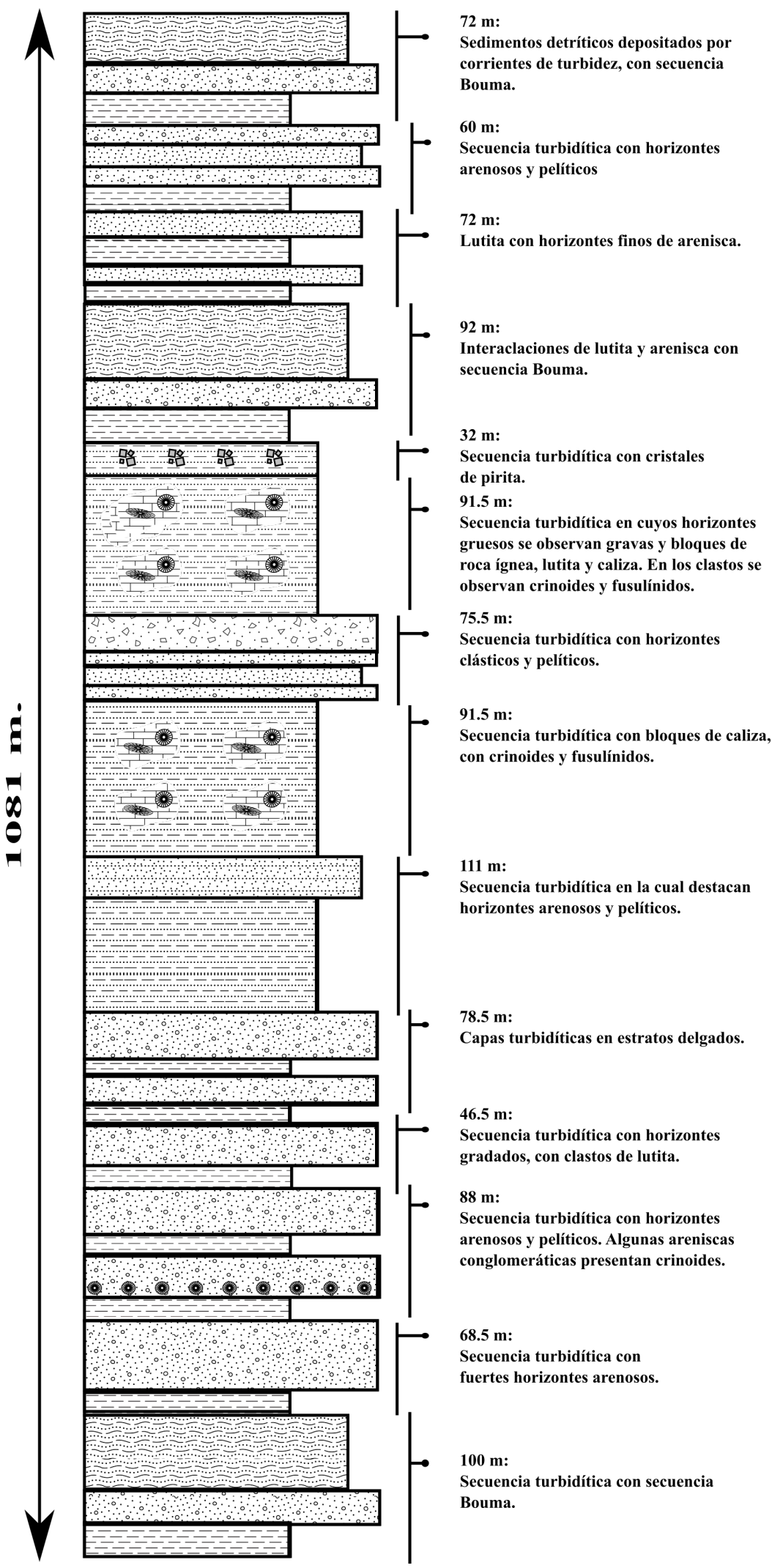


Unidad B: Secuencia turbidítica que consiste en una alternancia de horizontes arenosos de tonos claros con estratos de 10 y $20 \mathrm{~cm}$ de espesor y horizontes pelíticos de tonos oscuros estos últimos presentan espesores entre $20 \mathrm{~cm}$ y $1 \mathrm{~m}$. La unidad tiene un espesor total de $60 \mathrm{~m}$.

Unidad G: Secuencia de lutitas con horizontes finos de arenisca, dentro de los estratos de lutita se encontraron restos mal conservados de tallos de plantas, con estratos aproximados de 5 a $20 \mathrm{~cm}$ de espesor, las lutitas son de color gris claro y gris oscuro. La unidad cuenta con un espesor de $72 \mathrm{~m}$. Unidad D: Esta unidad presenta una intercalación de lutita y arenisca con secuencias Bouma de estratos entre 10 y $40 \mathrm{~cm}$ de espesor. La relación entre las fracciones de arenisca y lutita varían entre 1:1 y 2:1. La unidad tiene un espesor total de $92 \mathrm{~m}$. Unidad E: Secuencia flysch en estratos entre 5 y $20 \mathrm{~cm}$ con relación arenisca-lutita aproximada de 2:1. Las areniscas presentan los horizontes A, B, C y D de la secuencia Bouma, son de color gris claro, adquiriendo tonos ocres debido al intemperismo; contienen pequeños cristales de pirita, las lutitas son de color gris oscuro. La unidad tiene un espesor total de $32 \mathrm{~m}$.

Unidad F: Consiste en una secuencia flysch en cuyos horizontes gruesos se observan gravas y bloques de roca ígnea, lutita y caliza; en algunos clastos calcáreos se observan crinoides y fusulínidos, en tanto que, en otros se observan nódulos de pedernal; la unidad incluye estratos turbidíticos con secuencia Bouma completa. Por lo general, los estratos tienen espesores entre $60 \mathrm{~cm}$ y $1 \mathrm{~m}$, el espesor general de la unidad es de $91.5 \mathrm{~m}$.

Unidad G: En la parte basal de esta unidad se presenta una secuencia turbidítica con horizontes clásticos de tonos claros y horizontes pelíticos de tonos oscuros, aumentando hacia la parte superior la proporción de areniscas, los estratos tienen espesores entre $20 \mathrm{~cm}$ y $1 \mathrm{~m}$; la unidad incluye estratos con estructuras flasser y algunos diques de arenisca. El espesor de la unidad es de $75.5 \mathrm{~m}$.
Unidad H: La unidad inicia con estratos de tipo flysch con bloques de caliza con crinoides y fusulínidos. En la parte alta se observan estratos turbidíticos con un espesor de entre 20 y $50 \mathrm{~cm}$; son de color amarillo claro con secuencias Bouma completas. El espesor total de la unidad es de $91.5 \mathrm{~m}$. Unidad I: Secuencia flysch con horizontes de arenisca de color gris claro con estratos entre 20 y $50 \mathrm{~cm}$ de espesor; la parte pelítica es de color gris oscuro y estos estratos varían en espesor de 20 a 30 $\mathrm{cm}$. La unidad tiene un espesor general de $111 \mathrm{~m}$. Unidad J: Turbiditas en estratos delgados, los horizontes de arenisca tienen estratificación gradada, son de color café amarillento y presentan espesores entre 5 y $20 \mathrm{~cm}$, mientras que los de lutita son negros y sus espesores varían entre 4 y $10 \mathrm{~cm}$, el espesor total de la unidad es de $78.5 \mathrm{~m}$.

Unidad K: Secuencia turbidítica con horizontes gradados que incluyen una fracción conglomerática con clastos de lutita, estos horizontes tienen espesores aproximadamente de $60 \mathrm{~cm}$, mientras que el resto de los horizontes miden entre 13 y 20 $\mathrm{cm}$, la unidad es de color gris oscuro y cuenta con un espesor total de $46.5 \mathrm{~m}$.

Unidad L: Secuencia flysch con horizontes arenosos de aproximadamente $20 \mathrm{~cm}$ de espesor y horizontes pelíticos entre 1 y $2.6 \mathrm{~m}$ de espesor, algunas areniscas son conglomeráticas y presentan fragmentos de crinoides. La unidad incluye lentes de arenisca y diques sedimentarios, con un espesor total de $88.0 \mathrm{~m}$.

Unidad M: Secuencia compuesta de turbiditas de estratos delgados, con espesores de 10 a $15 \mathrm{~cm}$, en los cuales predomina el horizonte arenoso con gradación normal, estas rocas son de color gris oscuro y la parte pelítica es de color negro, teniendo la unidad un espesor de $68.5 \mathrm{~m}$.

Unidad N: Turbiditas de color gris oscuro con secuencias Bouma completas, cuyos horizontes arenosos tienen espesores entre 8 y $10 \mathrm{~cm}$, en tanto que los pelíticos son de aproximadamente $30 \mathrm{~cm}$, con un espesor de $100 \mathrm{~m}$. 


\section{Paleontología Sistemática}

Los fragmentos de las columnas y placas disociadas de los crinoides en los sustratos de Pemuxco son tan abundantes que forman acumulaciones (encrinita); no obstante, están mal conservados y en la mayoría de los casos no se pueden identificar, por lo cual se utilizó el método parataxonómico, propuesto por Moore y Jeffords (1968) y Jeffords y Miller (1968).

El material se encuentra depositado en la Colección Nacional de Paleontología del Instituto de Geología de la Universidad Nacional Autónoma de México, Ciudad Universitaria, Circuito Exterior, Delegación Coyoacán, CDMX, México; con los números de catálogo IGM-10152-IGM-10157.

Subclase y Orden incierta Moore y Jeffords, 1968 [Grupo Cyclici]

Familia Cyclomischidae Moore y Jeffords, 1968

Diagnosis. Crinoides que presentan generalmente, la columna heteromórfica, en algunos casos es homeomórfica. Las placas tienen la superficie articular con el crenulario ancho, el lumen circular, pero en casos aislados es pentalobado. Carecen de areola o perilumen o es insignificante (Moore y Jeffords, 1968, p. 58).

Género Cylindrocauliscus Moore y Jeffords, 1968

Cylindrocauliscus fiski Moore y Jeffords, 1968

Figura 3a

Cylindrocauliscus fiski Moore y Jeffords, 1968, p. 62, lám. 14, figs. 5a, b; Buitrón, 1977, p. 148, fig. 2; Moreno-Cano y Patiño Ruiz, 1981; Buitrón, Moreno-Cano y Patiño-Ruiz, 1987, p. 130, fig. 2; Buitrón y Solís, 1993, p. 214; Esquivel et al., 2000, p. 1 188; Buitrón et al., 2005, 2008.

Descripción. La placa articular es redonda, con el lumen circular, casi del ancho del crenulario y rodeado por un perilumen estrecho, ligeramente elevado. El crenulario está constituido por cúl- menes anchos, rectos y bifurcados generalmente antes de la mitad de su longitud; entre ellos hay escasos cúlmenes simples. Las crénulas son ligeramente más estrechas que los cúlmenes.

Ejemplar No. IGM-10152.

Dimensiones. La placa articular mide $5.3 \mathrm{~mm}$ de diámetro, el lumen $1.9 \mathrm{~mm}$ de ancho; el perilumen $1.1 \mathrm{~mm}$ y el crenulario $2.3 \mathrm{~mm}$ de ancho.

Discusión. Cylindrocauliscus fiski (Moore y Jeffords, 1968, p. 62) se describió por primera vez de la Lutita Mingus (Desmoinesiano), que pertenece al Grupo Strawn (Pensilvánico medio) de Texas, Estados Unidos de América.

Buitrón (1977, p. 148, fig. 2F) asignó a esta especie, ejemplares de crinoides recolectados en la Formación Santa Rosa Inferior (Pensilvánico inferior) cuyas rocas afloran en Chicomuselo-La Cioncordia en la región sureste del estado de Chiapas. Moreno-Cano y Patiño Ruiz (1981) y Buitrón et al. (1987, p. 130, fig. 2) describieron a Cylindrocauliscus fiski de la Formación del Monte (Pensilvánico) en la región de Calnali, estado de Hidalgo, Esquivel et al. (2000, p. 1188) del Paleozoico superior de las formaciones Olinalá, estado de Guerrero, Patlanoaya y Cuxtepeque, estado de Puebla; Buitrón et al. (2005; 2008) la citan de una secuencia de estratos del Pensilvánico de la Sierra Las Mesteñas, estado de Sonora y de la Formación Ixtaltepec, en Santiago Ixtaltepec, Oaxaca (Buitrón et al., 2008).

Género Baryschyr Moore y Jeffords, 1968

$$
\text { Baryschyr anosus Moore y Jeffords, } 1968
$$

Figura $3 \mathrm{~b}, 3 \mathrm{c}$

Baryschyr anosus Moore y Jeffords, 1968, p. 64, lám. 14, figs. 6-7; lám.15, figs. 7-11; lám.16, figs. 1-11; Moreno-Cano y Patiño Ruiz, 1981, p. 26, lám.2 fig. 4; lám. 3 fig. 3; Buitrón, Moreno-Cano y Patiño-Ruiz, 1987, p. 130, fig. 1; Buitrón y Solís, 1993, p. 214; Esquivel et al., 2000, p. 1188; Buitrón et al., 2005, 2008.

Descripción. La placa articular es circular con el lumen subcircular de contorno irregular, cuyas dimensiones corresponden a un tercio del diáme- 


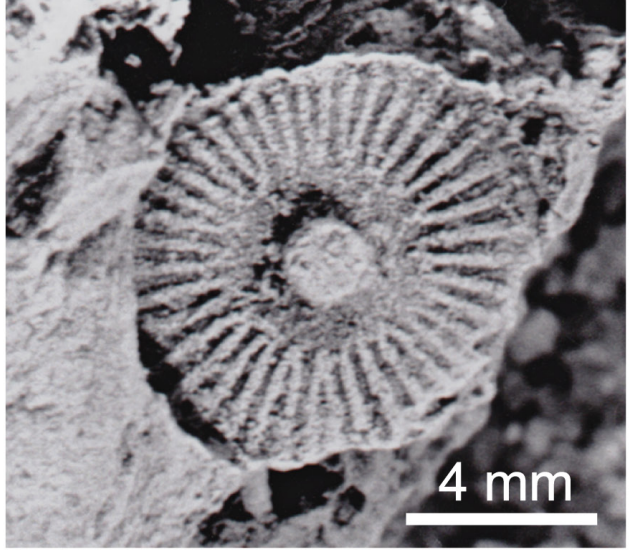

3a

$3 c$
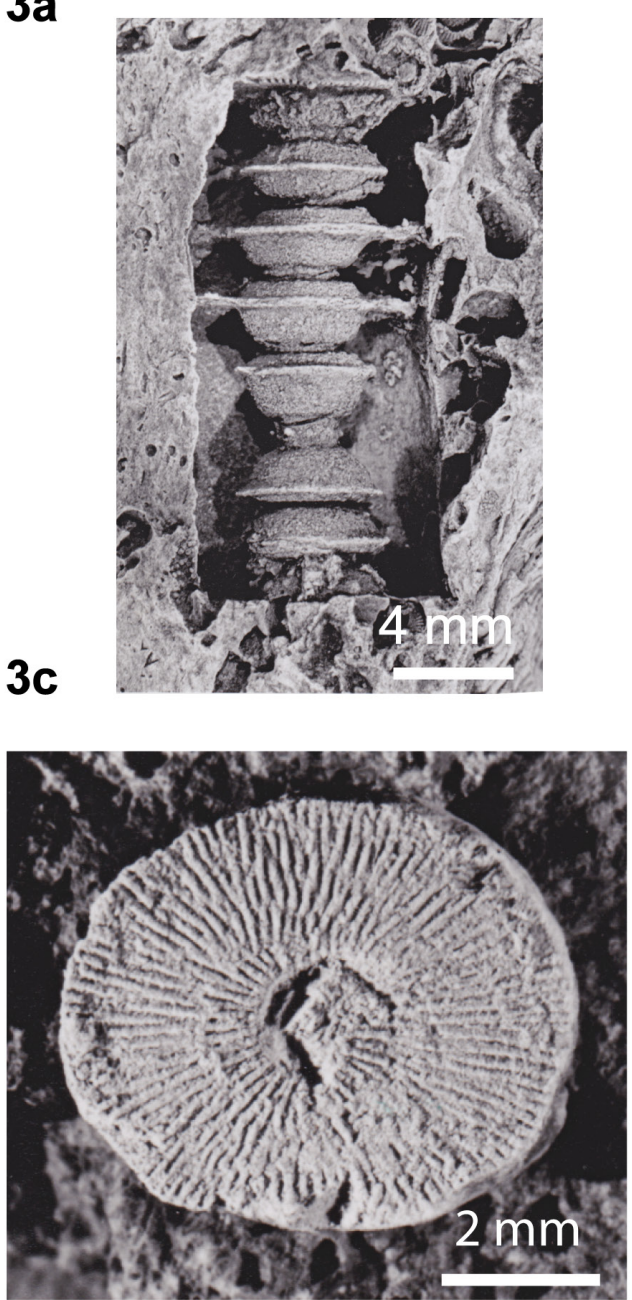

$3 e$

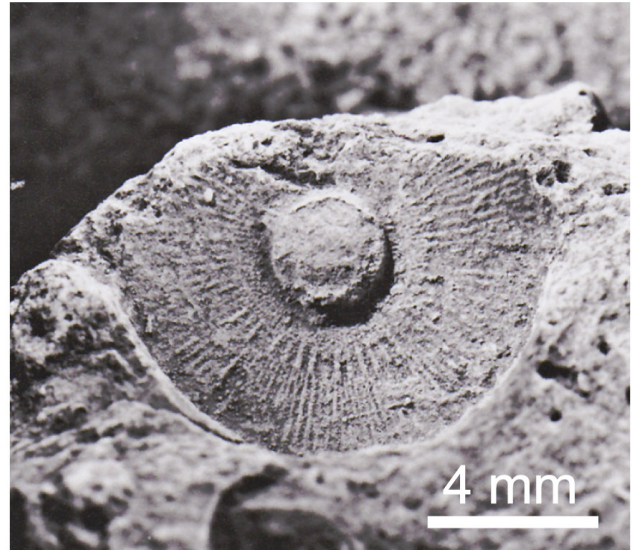

3b

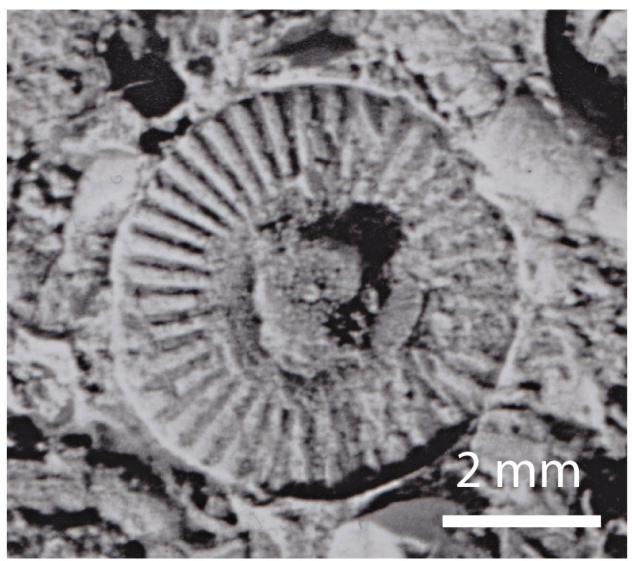

3d

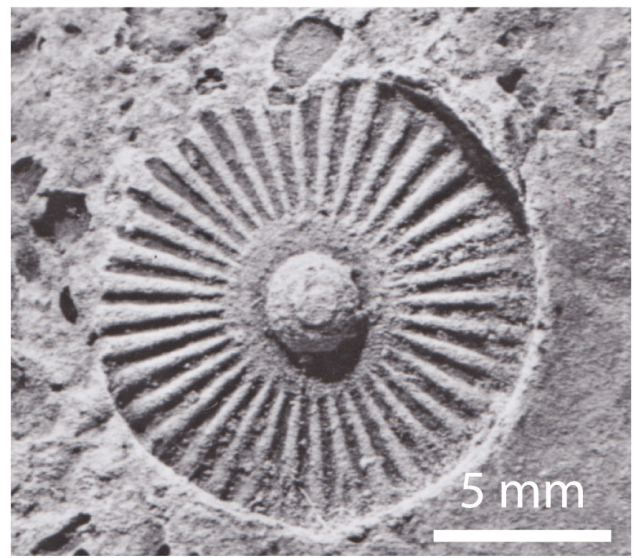

3f

Figura 3 Fotografías de las especies de crinoides descritas: (a) Cylindrocaulis cusfiski Moore y Jeffords, 1968, ejemplar IGM-10152, vista de la superficie articular de la placa (X7.6); (b) Baryschyr anosus Moore y Jeffords, 1968, ejemplar IGM-10153, vista de la superficie articular de la placa (X4.3); (c) Baryschyr anosus Moore y Jeffords, 1968, ejemplar IGM-10154, vista de un fragmento de la columna del crinoide (X3.0); (d) Cyclocaudex jucundus Moore y Jeffords, 1968, ejemplar IGM-10155, vista de la superficie articular de la placa (X6.2); (e) Cyclocaudex plenus Moore y Jeffords, 1968, ejemplar IGM-10156, vista de la superficie articular de la placa (X6.0); (f) Cycloscapus laevis Moore y Jeffords, 1968, ejemplar IGM-10157, vista de la superficie articular de la placa (X3.0). 
tro de la placa. No presenta perilumen ni areola. El crenulario es amplio, tiene cúlmenes y crénulas rectas, muy finas, éstas últimas ligeramente más estrechas que los cúlmenes. Un fragmento del relleno de la columna, está formado por seis placas donde se muestra un crenulario ancho.

Ejemplares No. IGM-10153-IGM-10154.

Dimensiones. La placa articular mide $10.2 \mathrm{~mm}$ de diámetro, el lumen $2.9 \mathrm{~mm}$ de ancho y el crenulario $7.3 \mathrm{~mm}$ de ancho.

Discusión. Moore y Jeffords (1968, p. 64) describieron la especie Baryschyr anosus del Pensilvánico medio de la Formación Dornick Hills, en la región sureste de Oklahoma, EUA. La especie fue citada por varios autores, entre ellos Moreno-Cano y Patiño Ruiz (1981), Buitrón, Moreno-Cano y Patiño-Ruiz (1987, p. 130, fig. 1) del Pensilvánico de Calnali, estado de Hidalgo y por Esquivel et al. (2000, p. 1188) de varias localidades del Paleozoico superior del sur de México.

Género Cyclocaudex Moore y Jeffords, 1968

Cyclocaudex jucundus Moore y Jeffords, 1968 Figura 3d

Cyclocaudex jucundus Moore y Jeffords, 1968, p. 66, lám. 17, figs. 6-7; Moreno-Cano y Patiño Ruiz, 1981, p. 26, lám. 2, fig. 3; Buitrón, Moreno-Cano y Patiño-Ruiz 1987, p. 132, fig. 5; Esquivel et al., 2000, p. 1188; Buitrón et al., 2012, p. 4).

Descripción. La placa articular es de contorno circular, la cicatriz del lumen es pequeña, con perilumen saliente, estrecho, rodeado de una areola ancha deprimida, provista de tubérculos muy finos. El crenulario ocupa la mitad de la placa y tiene cúlmenes medianamente gruesos, rectos y simples.

Ejemplar No. IGM-10155.

Dimensiones. La placa articular mide $8.8 \mathrm{~m}$ de diámetro, el lumen $1.4 \mathrm{~mm}$ de ancho, la areola 1.7 $\mathrm{mm}$ de ancho y el crenulario $5.7 \mathrm{~mm}$ de ancho.

Discusión. Las placas columnares de los ejemplares de la especie Cyclocaudex jucundus procedentes de Pemuxco, Hidalgo son abundantes, pero en general están mal conservadas. Sin embargo, son reconocidas por la presencia del perilumen saliente, la areola con granulaciones y el crenulario que presenta cúlmenes rectos, ligeramente anchos. La especie Cyclocaudex jucundus ha sido descrita por Moore y Jeffords (1968, p. 66, lám. 17, fig. 6-7) de la Formación Thrifty del Pensilvánico superior de Texas, EUA.

También ha sido citada de las rocas de la Formación del Monte (Pensilvánico superior) de la región de Calnali, Hidalgo, México (Moreno-Cano y Patiño Ruiz, 1981, p. 26 lám. 2, fig.3; Buitrón et al. (1987, p. 132, fig. 5). Esquivel et al. (2000) la informan del Paleozoico superior de las formaciones Patlanoaya, Puebla y Olinalá, Guerrero. Buitrón et al. (1998) del Cañón de La Peregrina, Tamaulipas y Buitrón et al. (2012) de Cerros El Tule, estado de Sonora.

\section{Cyclocaudex plenus Moore y Jeffords, 1968} Figura $3 \mathrm{e}$

Cyclocaudex plenus Moore y Jeffords, 1968, p. 66, lám. 18, figs. 1-5; Moreno-Cano y Patiño Ruiz, 1981, lám. 2, fig. 1; Esquivel et al., 2000, p. 1188; Buitrón et al., 2008, p. 4; Buitrón et al., 2012, p. 14. Descripción. La placa articular es circular, el lumen es redondo y pequeño, con areola estrecha. El crenulario presenta cúlmenes finos, rectos casi en su totalidad, algunos bifurcados en el tercio interno de la superficie articular; los cúlmenes son más anchos que las crenulaciones.

Ejemplar No. IGM-10156.

Dimensiones. La placa articular mide $12 \mathrm{~mm}$ de diámetro, el lumen $4.1 \mathrm{~mm}$ de ancho y el crenulario $8.4 \mathrm{~mm}$ de ancho.

Discusión. Cyclocaudex plenus presenta dos características morfológicas que permitieron su identificación: el lumen y la superficie areolar muy pequeños. Esta especie fue descrita por Moore y Jeffords (1968, p. 66, lám. 18, figs.1-5) de la Formación Thrifty y de la Formación Graham ambas del Pensilvánico superior de Texas, EUA. En el Paleozoico de México ha sido citada anteriormente de Calnali (Moreno-Cano y Patiño Ruiz, 1981, p. 
26, lám. 2 fig. 1), de Pemuxco, Hidalgo (Buitrón et al., 2008; Buitrón et al., 2012) y de Sierra Las Mesteñas (Buitrón et al., 2005).

Familia Leptocarphiidae Moore y Jeffords, 1968

Diagnosis. Columna heteromórfica, delgada a gruesa, de contorno circular y lados rectos. La faceta articular tiene el lumen amplio, propensa a formar un perilumen, nódulos comúnmente con cirros (Moore y Jeffords, 1968, p. 79).

Género Cycloscapus Moore y Jeffords, 1968

Cycloscapus laevis Moore y Jeffords, 1968

Figura $3 \mathrm{f}$

Cycloscapus laevis Moore y Jeffords, 1968, p. 83, lám. 27, figs. 15a, b; Buitrón et al., 2008, p. 4, tabla 1.

Descripción. La placa articular es de contorno circular, el lumen es grande, con perilumen incipiente y con granulaciones. La faceta articular es ligeramente de mayor tamaño que el lumen y está formada por cúlmenes rectos, gruesos y anchos, separados por crénulas profundas y anchas.

Ejemplar No. IGM-10157.

Dimensiones. La placa articular mide $8.8 \mathrm{~mm}$ de diámetro, el lumen $1.4 \mathrm{~mm}$ de ancho, el perilumen $2.0 \mathrm{~mm}$ de ancho y el crenulario $5.7 \mathrm{~mm}$ de ancho.

Discusión. Cycloscapus laevis fue descrita por primera vez por Moore y Jeffords (1968, p. 83, lám. 27, figs. 15a b) del Pensilvánico medio (Desmoinesiano) de la Lutita Mingus del Grupo Strawn en Texas, EUA. También ha sido citado para el Paleozoico Superior de la Sierra Agua Verde (Buitrón et al., 2008, p. 4, tabla 1). El ejemplar procedente de Pemuxco, Hidalgo, está bien conservado y muestra características afines a la especie Cycloscapus laevis Moore y Jeffords, 1968, no obstante, el ejemplar de Pemuxco, Hidalgo presenta un perilumen con gránulos pequeños.

\section{Consideraciones Bioestratigráficas}

En el área estudiada no se observa la base de la Formación Tuzancoa, pero se ha reportado al noreste un contacto discordante con el Gneis Huiznopala, sobre el río Jalapa (Ochoa-Camarillo, 1996, 1997). El contacto superior de la Formación Tuzancoa es discordante con los lechos rojos de la Formación Huizachal, tal como se observa en los cortes de la terracería de Jalapa, Hidalgo (Hoja Zacualtipán, INEGI, 2001).

La Formación Tuzancoa contiene a las especies de crinoides Cylindrocauliscus fiski, Baryschyr anosus, Cyclocaudex jucundus, Cyclocaudex plenus y Cycloscapus laevis descritas de otras localidades del Paleozoico superior de Texas y Oklahoma en Estados Unidos de Norteamérica (Moore y Jeffords, 1968), asociadas a los foraminíferos de los géneros Boultonia, Lunacammina y Globivalvulina, Schwagerina y Triticites del Paleozoico superior y particularmente del fusulínido Skinerella que ha sido citado de las Montañas Glass, de las Montañas Hess y de la Sierra del Diablo en Texas, EUA y también anteriormente han sido reportados de varias localidades de México (Hidalgo, Chiapas), Guatemala y Belice (Arellano et al., 1998; Vachard et al., 2004; Buitrón et al., 2008) que precisa la edad del Pérmico temprano (Leonardiano) para toda la asociación fósil. Además, se hallaron fragmentos de briozoarios fenestélidos y braquiópodos espiriféridos. La flora fósil consiste en algas del género Tubiphytes y Epimastopora así como tallos de plantas mal conservados (Silva, 1987; Vachard et al., 1997; Arellano et al., 1998).

\section{Consideraciones Paleoecológicas}

La asociación fósil de la región de Pemuxco está conformada por algas del género Tubiphytes y Epimastopora, por pequeños foraminíferos de los géneros Diplosphaerina/Eotuberitina, Earlandia, Endothyra, 
Tetrataxis, Climacammina, Globivalvulina, Calcitornella, "Arenovidalina", Nodosaria, Geinitzina y fusulínidos de los géneros Staffella y Skinnerella, fragmentos de briozoarios fenestélidos, braquiópodos espiriféridos (Vachard et al., 1997; Arellano et al., 1998) y crinoides de las especies Cylindrocauliscus fiski Moore y Jeffords, 1968, Baryschir anosus Moore y Jeffords, 1968, Cyclocaudex jucundus Moore y Jeffords, 1968, Cyclocaudex plenus Moore y Jeffords, 1968, Cycloscapus laevis Moore y Jeffords, 1968.

La macroflora fósil depositada en los estratos de la Formación Tuzancoa consiste en tallos de plantas vasculares mal conservadas (Silva, 1987). Los crinoides se desarticularon con facilidad al morir y los restos son tan abundantes, que no se puede hablar del número preciso de individuos o de su relación con la cantidad de taxones, desde el punto de vista de los índices de diversidad, ni desde el enfoque de la sucesión de comunidades, lo que equivale a considerar que a pesar de su manifiesta abundancia (encrinita) no se reconocen parámetros de poblaciones o de comunidades cuantificables. Se infiere que la gran cantidad de nutrientes determinaba la abundancia y diversidad de la mayoría de las especies (Moore, 1938; Meyer, 1971; Meyer y Macurda, 1974; Menn, 1994).

Los crinoides procedentes de la región de Pemuxco probablemente vivieron en un ambiente batial de facies flysch, que corresponden a un depósito rítmico presente en la Formación Tuzancoa y que sugiere agua relativamente poco profunda, en un ambiente geotectónico inestable, con el aporte de sedimentos acarreados por corrientes de turbidez que erosionaron y seleccionaron los clastos, posiblemente provenientes de la plataforma continental, generando lo que tiempo más tarde al consolidarse sería la alternancia de flysch y secuencias Bouma (Vachard et al., 1997; Arellano et al., 1998; Rosales-Lagarde et al., 2005). Es importante hacer mención de que en este periodo ocurre la Revolución Apalachiana, Maratón Ouachita y Huastecana, que propició la elevación continental y orogenia en Norte América y Centroamérica.
Las variaciones verticales en la secuencia medida en el río Tianguistengo, indican una acumulación turbidítica cuyas facies primero fueron proximales y después distales, las intercalaciones de conglomerado con bloques compuestos de fusulínidos y crinoides sugieren la denudación de una plataforma calcárea y el transporte subacuoso de sus fragmentos hacia las zonas más profundas. Así la unidad se acumuló tanto en el talud continental como en la planicie abisal, en el marco tectónico de la desintegración del gran continente Pangea.

\section{Conclusiones}

Se describe cinco especies de crinoides: Cylindrocauliscus fiski Moore y Jeffords, 1968, Baryschir anosus Moore y Jeffords, 1968, Cyclocaudex jucundus Moore y Jeffords, 1968, Cyclocaudex plenus Moore y Jeffords, 1968, Cycloscapus laevis Moore y Jeffords, 1968, procedentes de afloramientos de la Formación Tuzancoa del Pérmico (Leonardiano) en la región de Pemuxco, en el estado de Hidalgo. La presencia en dichos afloramientos del fusulínido Skinnerella, en el mismo nivel que los crinoides, precisa la edad del Pérmico inferior (Leonardiano) para esta secuencia.

Los crinoides procedentes de la región de Pemuxco probablemente vivieron en un ambiente batial de facies flysch, que corresponden a un depósito rítmico presente en la Formación Tuzancoa y que sugiere agua relativamente poco profunda, en un ambiente geotectónico inestable.

Los crinoides han sido citados de otras localidades del Paleozoico superior de México (Calnali, Hidalgo; Patlanoaya y Cuxtepeque Puebla; Ixtaltepec, Oaxaca, Cañón de la Peregrina, Tamaulipas, Cerros El Tule, Sierra Agua Verde y Sierra Las Mesteñas, Sonora. En los Estados Unidos de Norteamérica se describieron de Texas (Lutita Mingus, Grupo Strawn; Formación Thrifty, Formación Graham) y de Oklahoma (Caliza Marble Falls). 


\section{Agradecimientos}

Los autores agradecen el apoyo de los Proyectos CONACyT No. 165826, UNAM-DGAPA-PAPIIT No. 105012 y ECOS No. M13U01 para realizar la presente investigación. Asimismo, a los Ingenieros Geólogos Héctor Camarillo Ochoa y Javier Arellano Gil que proporcionaron la asesoría en campo sobre la prospección geológico-paleontológica en diversas ocasiones en afloramientos del Anticlinorio de Huayacocotla, en los estados de Tamaulipas e Hidalgo. El M. en C. José Carlos Jiménez López realizó las ilustraciones.

\section{Referencias}

Arellano, G.J., Vachard, D., Yussim, S., Flores de Dios, A., 1998, Aspectos estratigráficos, estructurales y paleogeográficos del Pérmico Inferior al Jurásico Inferior en Pemuxco, Estado de Hidalgo, México: Revista Mexicana de Ciencias Geológicas, 15(1), 9-13.

Brunner, P., 1984, Catálogo de microfósiles índice del Paleozoico, Jurásico Superior-Cretácico y microfacies del Paleozoico de México, parte I: Fusulinácea: Instituto Mexicano de Petróleo, no paginado.

Buitrón, B.E., 1977, Invertebrados (Crinoidea y Bivalvia) del Pensilvánico de Chiapas. Universidad Nacional Autónoma de México: Revista del Instituto de Geología, 1(2), 144-150.

Buitrón, B.E., Moreno-Cano, A., Patiño-Ruiz, J., 1987, Crinoides del Paleozoico Tardío (Pensilvánico) de Calnali, Hidalgo: Revista de la Sociedad Mexicana de Paleontología, 1(1), 125-136.

Buitrón-Sánchez, B.E., Solís-Marín, F. A., 1993, La biodiversidad de los Equinodermos fósiles y recientes de México: Revista de la Sociedad Mexicana de Historia Natural, Volumen Especial XLIV, 2009-2031.
Buitrón-Sánchez, B.E., Arellano-Gil, J., Flores de Dios, L.A., 1998, Crinoides del Pensilvánico del Cañón de la Peregrina, Estado de Tamaulipas, México en Primera Reunión Nacional de Ciencias de la Tierra: México D.F., México, Unión Geofísica Mexicana, Resúmenes, 55 p.

Buitrón-Sánchez, B.E., Almazán-Vázquez, E., Vachard, D., 2005, Los crinoides pensilvánicos de los cerros Las Mesteñas en la región nororiental del estado de Sonora y sus implicaciones paleogeográficas: GEOS, 25(1), 151-152.

Buitrón-Sánchez, B.E., Gómez-Espinosa C., Almazán-Vázquez, E., Vachard, D., Laguarda-Figueras, A., Solís-Marín, F., 2008, A review of the crinoid columnals (Echinodermata-Crinoidea) from the Carboniferous of Mexico: Revista Biología Tropical, 56(3), 1-12.

Buitrón-Sánchez, B.E., Vachard, D., AlmazánVázquez, E., Palafox, J.J., 2012, Una Secuencia Cratónica Completa del Carbonífero al Pérmico Inferior, expuesta en Los Cerros El Tule, en el noreste de Sonora, noroeste de México: Revista Mexicana de Ciencias Geológicas, 29(2), 39-62.

Campa, H.F., Coney, P.J., 1983, Tectonostratigraphic terranes and mineral resource distributions in Mexico: Canadian Journal of Earth Sciences, 20, 1040-1051.

Carrillo-Bravo, J., 1959, Notas sobre el Paleozoico de la región de Ciudad Victoria, Tamaulipas: Boletín de la Asociación Mexicana de Geólogos Petroleros, 1(11-12), 671-680.

Carrillo-Bravo, J., 1961, Geología del Anticlinorio Huizachal-Peregrina al NW de Cd. Victoria, Tamaulipas: Boletín de la Asociación. Mexicana de Geólogos Petroleros, 13(1-2), $1-98$.

Carrillo-Bravo, J., 1965, Estudio Geológico de una parte del Anticlinorio de Huayacocotla.: Boletín de la Asociación Mexicana de Geólogos Petroleros, 17(5-6), 73-96. 
Esquivel, M.C., Ausich, A., Buitrón, B.E., Flores de Dios, A., 2000, Pennsylvanian and Mississippian pluricolumnal assemblages (Class Crinoidea) from Southern Mexico and new occurrence of a column with tetralobate lumen: Journal of Paleontology, 74(6), 1187-1190.

INEGI, 2001, Hoja Zacualtipán F14D62, Hidalgo y Veracruz, Carta topográfica 1:50000, disponible en <http://www.inegi.gob.mx> .

Jeffords, R.S., Miller, T.H., 1968, Ontogenic development in late Pennsylvanian crinoid columnals and pluricolumnals: University of Kansas and Soc. Geol. of America, p. 1-14.

Kuegelgen, H., Von, 1958, Der Aufbau der zentralen Sierra Madre Oriental, México: Zeitschr. Deutsch. Geol. Gesellschaft, 110, 117-142.

Martínez-Pérez, P., 1962, Estudio geológico de una porción de la Sierra Madre Oriental al oriente de Zacualtipan y Tianguistengo, Hidalgo: Instituto Politécnico Nacional, Escuela Superior de Ingeniería y Arquitectura (ESIA), Ciencias de la Tierra, Tesis de Licenciatura, $48 \mathrm{p}$.

Menn,J., 1994, La reconstitution des communautés de crinoides paléozoïques: Lópport des columnals disocies, en David, B., Guille, A., Féral, J.-P., and Roux, M. (eds.) Proceedings of the $8^{\text {th }}$ International Echinoderm Conference, Dijon, France, 6-10 September 1993. Balkema, Rotterdam/Brookfield, 231-236.

Meyer, D.L., 1971, Post mortem disarticulation of recent crinoids and Ophiuroids under naturalconditions: The Geological Society of America, Abstracts, 3(7), 645-646.

Meyer, D.L., Macurda, D.B., 1974, The zoogeography of Caribbean crinoids; a model for paleontological interpretation. Geological Society of America, Abstracts, 6(7), 868.

Moore, R.G., 1938, The use of fragmentary crinoidal remains in stratigraphic paleontology: Bulletin of the Scientific
Laboratories of Denison University 38(10), 165-250.

Moore, R.C., Jeffords, R.S., 1968, Classification and nomenclature of fossil crinoids based on studies of dissociated parts of their columns: University of Kansas, Paleontological Contributions, Echinodermata, 9-86.

Moreno-Cano, L.A., Patiño Ruiz J., 1981, Estudio del Paleozoico en la región de Calnali, Hidalgo: Ciudad de México, Instituto Politécnico Nacional, Escuela Superior de Ingeniería y Arquitectura (ESIA), Tesis de Licenciatura, 64 p.

Ochoa-Camarillo, H., 1996, Geología del Anticlinorio de Huayacocotla en la región de Molango, Estado de Hidalgo: Universidad Nacional Autónoma de México, Facultad de Ciencias, División de Estudios de Posgrado, Tesis de Maestría en Ciencias (Geología), 91 p.

Ochoa-Camarillo, H., 1997, Geología del Anticlinorio de Huayacocotla en la región de Molango, Hidalgo, en II Convención sobre la Evolución Geológica de México y Recursos Asociados: Pachuca, Hidalgo, Instituto de Investigaciones en Ciencias de la Tierra e Instituto de Geología, Hidalgo y UNAM: Simposio y Coloquio, 1-17.

Ochoa-Camarillo, H., Buitrón, B.E., Silva Pineda, A., 1997, Red beds of the Huayacocotla Anticlinorium, State of Hidalgo, east-central Mexico: Geological Society of America Abstracts, 50(2), 42.

Pérez-Ramos, O., 1979, Estudio estratigráfico del Paleozoico Superior en el río Chinameca, Hidalgo: Instituto Mexicano del Petróleo, Proyecto-3039, 27 p. (Inédito).

Rosales-Lagarde, L., Centeno-García, E., OchoaCamarillo, H., Sour-Tovar, F., 1997, Permian volcanism in Eastern Mexico-preliminary report: Instituto de Investigaciones en Ciencias de la Tierra e Instituto de Geología, Universidad Nacional Autónoma de México, II Convención sobre la Evolución Geológica de México y Recursos Asociados, Pachuca, 
Hidalgo, Simposio y Coloquio, 31-41.

Rosales-Lagarde, L., Centeno-García, E., Dostal, J., Sour-Tovar, F., Ochoa-Camarillo, H. Quiroz-Barroso, S., 2005, The Tuzancoa Formation: Evidence of an Early Permian Submarine Continental Arc in East-Central Mexico: International Geology Review, 47(9), 901-919.

Silva-Pineda, A., 1987, Algunos elementos paleoflorísticos del Pérmico de la región de Calnali, Estado de Hidalgo: Revista de la Sociedad Mexicana de Paleontología, 1(1), 313-327.

Stinnesbeck, W., 1994, Icnofósiles de la Formación Guacamaya (Pérmico Inferior) del Cañón La Peregrina, al noreste de Ciudad Victoria, Tamaulipas, México: Revista de la Sociedad Mexicana de Paleontología, 7(2), 47-55.
Vachard, D., Grajales, J.M., Flores de Dios, A., Torres, R., Buitrón, B.E., 1997, Patlanoaya and Juchatengo: Two key sequences for under standing the late Paleozoic geological history of Mexico, en II Convención sobre la Evolución Geológica de México y Recursos Asociados: Pachuca, Hidalgo, Instituto de Investigaciones en Ciencias de la Tierra e Instituto de Geología, Hidalgo y UNAM: Simposio y Coloquio, 37-41.

Vachard, D., Flores de Dios, A., Buitrón B., 2004, Guadalupian and Lopingian (Middle and Late Permian) deposits from México and Guatemala, a review with new data: GEOBIOS 37, 99-115. 